\title{
Coordenação de Veículos Aéreos não Tripulados Utilizando Inteligência de Enxames
}

Luiz Felipe F. de Oliveira

Escola Politécnica de Pernambuco

Universidade de Pernambuco

50.720-001 - Recife, Brasil
Diego M. P. F. Silva

Escola Politécnica de Pernambuco

Universidade de Pernambuco

50.720-001 - Recife, Brasil
Carmelo J. A. Bastos Filho

Escola Politécnica de Pernambuco Universidade de Pernambuco 50.720-001 - Recife, Brasil Autor2@meu-email

Resumo Este artigo apresenta a análise de um modelo para a coordenação de veículos aéreos não tripulados (UAVS) utilizando uma abordagem de inteligência de enxames baseado no Particle Swarm Optimization. O modelo considera mecanismos de locomoção e anticolisão, comunicação ad hoc e informações do ambiente obtidas dos sensores do UAV. Foi analizada a performance em termos de rastreamento de alvos e escalabilidade. Um simulador foi desenvolvido com uma interface gráfica para permitir uma n'alise visual do enxame de UAVs.

Palavras-Chave: Inteligência de enxames; Modelos bio-inspiradores 


\section{Introdução}

Os Veículos Aéreos não Tripulados (UAVs, Unmanned Aerial Vehicles) têm recebido bastante interesse da comunidade científica nos últimos anos e, graças aos investimentos em computação embarcada, telecomunicações, sensores e redução de consumo de energia, estes estão cada vez mais capazes de desempenhar tarefas bastante complexas e sofisticadas. Como não são ocupados por nenhum humano, os UAVs são geralmente mais simples e de menor custo quando comparados com outros veículos aéreos, sendo adequados para missões longas e perigosas, como monitoramento de tráfico urbano, operações militares de busca e salvamento e patrulha de fronteira [1]. Existem váriios tipos de UAVs para diferentes propósitos, como os exemplos mencionados anteriormente [2].

Inteligência de enxames (Swarm Intelligence) [3] [4] [5], que é um paradigma de inteligência computacional que utiliza inspiração biológica e é baseado em populações foi utilizada como abordagem na coordenação dos múltiplos UAVs. Tal abordagem possui ênfase em distributividade, interação direta e indireta, flexibilidade e robustez.

Para permitir que os UAVs se engajem em missões de longa duração, não tendo que ser recarregados frequentemente, o consumo de energia não deve ser alto. Dessa forma, uma comunicação de longo alcance, que por sua vez requer transmissores de alta potência, se torna inviável por conta do consumo de energia. Esse problema pode ser resolvido utilizando um paradigma de comunicação ad hoc, onde todos os UAVs podem funcionar como ponte de roteamento, ou seja, ao invés de transmitir a informação diretamente para o destino, a informação é transmitida para o UAV imediatamente vizinho, e assim sucessivamente até que a informação chegue ao destino.

Com o objetivo de analisar o comportamento dos enxames de robôs, uma ferramenta computacional que simula a inspeção de dutos submarinos utilizando inteligência de enxames em robôs aquáticos foi proposta em [6]. Este estudo mostra que múltiplos robôs podem realizar a inspeção de forma mais efetiva que um único robô e que, dependendo das características do ambiente, aumentar demasiadamente o número de robôs não gera benefícios adicionais relevantes, somente custos.

Um simulador de UAVs utilizando inteligência de enxame e considerando os aspectos de comunicação, colisão, afastamento e rastreamento de alvos auxilia também a guiar o projeto e configuração do enxame necessário para implantação real em campo. O relato de algumas experiências com UAVs, inclusive de implementação prática, podem ser verificados em [1] [7] [8]. Entretanto, nenhum desses trabalhos propôs um mecanismo de coordenação autônomo de um enxame de UAVs considerando colisão, afastamento, comunicação ad hoc e rastreamento de alvos m'oveis ao mesmo tempo.

O restante do artigo está organizado da seguinte forma: o conceito de inteligência de enxames é introduzido na Seção II, os conceitos de enxame de robôs são apresentados na Seção III, os mecanismos e o comportamento do sistema proposto são apresentados na Seção IV, os resultados das simulações são apresentados na Seção V e as conclusão e trabalhos futuros são apresentados na Seção VI.

\section{Fundamentos de Inteligência de En- xames e Otimização por Enxame de partículas}

A metáfora de insetos sociais para solução de problemas se tornou uma linha de pesquisa em alta nos últimos 15 anos, visto que essa abordagem coloca em ênfase aspectos como distributividade, interação direta e indireta entre agentes relativamente simples, flexibilidade e robustez [9].

Inteligência de enxames teve sua origem a partir de estudos sobre colônias e enxames de organismos sociais simples. Um enxame pode ser descrito como uma coleção estruturada de organismos interativos (ou agentes), onde cada indivíduo é extremamente simples e o comportamento coletivo apresentado se torna bastante complexo. Este comportamento global do enxame emerge de maneira não linear através do comportamento dos indivíduos, existindo assim um forte acoplamento entre o comportamento individual e o comportamento do enxame. Dessa forma, o comportamento coletivo dos indivíduos especifica o comportamento do enxame e a interação entre indivíduos desempenha um papel fundamental para a emergência desse comportamento [3].

Os estudos em enxames de organismos sociais levaram à construção de algoritmos de otimização e agrupamento bastante eficientes [4], como a Otimização por enxames de partículas (PSO, Particle Swarm Optimization) e a Otimização por colônias de formigas (ACO, Ant Colony Optimization) [3].

O PSO, desenvolvido em 1995 por J. Kennedy e R. Eberhart, é uma técnica de otimização estocástica desenvolvida inicialmente com o intuito de simular graficamente o comportamento de um bando de pássaros [5].

Um aspecto que guia o PSO é a interação social, onde indivíduos dentro do enxame aprendem uns com os outros e, baseado no conhecimento obtido, cada indivíduo move- 
se com o objetivo de tornar-se mais próximo de seus melhores vizinhos. Tal estrutura social é determinada pela formação de vizinhanças, onde os indivíduos dentro dos limites dessa Vizinhança, comunicam-se uns com os outros.

Existem diferentes tipos de vizinhanças que foram definidas e estudadas, como por exemplo: topologia em estrela (star topology) [10], onde cada partícula pode comunicar-se com qualquer outra dentro do enxame, formando um rede social totalmente conectada; e a topologia em anel (ring topology) [10], onde cada partícula comunica-se com $\mathrm{o}$ seus $\mathrm{n}$ vizinhos imediatos e o valor de $\mathrm{n}$ é um inteiro não negativo menor que o tamanho do enxame e maior que 1 (um). A noção de vizinhança é baseada no rótulo das partículas.

Em um enxame, cada partícula representa uma solução potencial. As partículas voam pelo hiperespaço, modificando seu posicionamento baseado em sua própria experiência e nas experiência de seus vizinhos. Cada partícula armazena as seguintes informações:

\section{Fundamentos de Enxames de Robôs}

Enxame de robôs é uma nova abordagem para coordenação de um grande número de robôs relativamente simples, autônomos, não controlados de maneira centralizada, capazes de se comunicar localmente e que operam baseados em inspiração biológica [11]. Inteligência de enxames de robôs pode ser entendida como uma propriedade de um sistema de robôs não inteligentes exibindo coletivamente um comportamento inteligente [12]. A aplicação dos princípios de inteligência de enxames em uma coleção de robôs pode ser denominada como enxame de robôs (Swarm robotics) [11].

Segundo [13], um enxame de robôs deve apresentar três propriedades funcionais que são observadas em enxames naturais e permanecem como propriedades desejadas em um sistema multi-robôs. São elas:

1. Robustez: capacidade de continuar suas operações apesar de distúrbios no ambiente ou falha de algum indivíduo

2. Flexibilidade: indivíduos com a habilidade de coordenar seus comportamentos de modo que sejam capazes de enfrentar tarefas de diferentes naturezas.

3. Escalabilidade: capacidade de operar com grupos de tamanho variados e em um amplo intervalo, suportando também um número demasiado grande de indivíduos sem gerar impacto considerável no desempenho.
Dentre essas propriedades, os maiores interesses nas pesquisas são a resolução de problemas de cobertura e exploração de ambientes de maneira eficiente, controle distribuído, difusão e interação.

\section{Mecanismos do sistema proposto}

Os UAVs foram modelados nesta pesquisa como agentes reativos simples com suas respectivas propriedades, percebendo o ambiente a partir de sensores, processando sobre as informações obtidas desses mesmos sensores e atuando no ambiente.

O processo de coordenação é similar ao processo de compartilhamento de informação do PSO. Esse artigo propõe uma adaptação do algoritmo de otimização PSO para ser utilizado na coordenação dos UAVs. Para a realização das atividades de patrulha e rastreamento, os UAVs devem possuir alguns mecanismos que serão descritos a seguir.

\section{A. Mecanismos de Comunicação}

A comunicação utilizada em robôs móveis, geralmente, é sem fio devido à característica dinâmica do ambiente. Embora existam UAVs que utilizam sistema de comunicação via satélite [14], neste simulador foi proposto um sistema de comunicação sem fio com uma topologia ad hoc, ou seja, cada UAV é uma ponte de roteamento. É importante observar que o sensor de comunicação influencia a locomoção do UAV com o objetivo de evitar a perda de comunicação.

\section{B. Mecanismos de Localização}

Os UAVs possuem o objetivo de patrulhar o ambiente em que se encontram e rastrear eventuais alvos. Os UAVs podem armazenar uma representação do ambiente em que se encontram por meio de um mapa ou apenas possuir o conhecimento de sua localização no ambiente. No modelo proposto, cada UAV possui o conhecimento de sua localização no ambiente o que o possibilita locomover-se no ambiente, tomar decisões, retornar à base, evitar colisões e sair de seu espaço aéreo, rastrear possíveis 'alvos e atuar de maneira a manter-se conectado aos seus vizinhos.

\section{Mecanismo anti-colisão}

O UAV possui um sensor anti-colisão para possibilitar uma locomoção segura através do ambiente. Obstáculos podem ser entendidos como, por exemplo, estruturas fixas e outros UAVs.

D. Mecanismo de percepção do ambiente 
O sensor de percepção ambiental é utilizado para possibilitar a detecção de alvos no ambiente e permitir que o UAV inicie o rastreamento dinâmico desses alvos.

\section{E. Mecanismo de locomoção}

O sensor de percepção ambiental é utilizado para possibilitar a detecção de alvos no ambiente e permitir que o UAV inicie o rastreamento dinâmico desses alvos.

- Sincronismo: Com o objetivo de atuar no sentido de afastamento para evitar colisões ou no sentido de aproximação para evitar a perda de comunicação;

- Cognição: Com o objetivo de patrulhar de maneira estocástica ou com o objetivo de perseguir e comunicar as posições dos alvos dependendo do estado em que se encontra;

\section{Resultados}

Para as simulações foram utilizados três conjuntos experimentais, descritos a seguir:

\section{A. Conjunto experimental 1}

Tem como objetivo analisar a escalabilidade do modelo proposto em função do número de UAVs utilizando 5 alvos e observando as seguintes métricas:

- CL: Percentual de colisões ocorridas. Essa métrica é calculada pela diferença da quantidade de UAVs no início com a quantidade de UAVs no fim em relação à quantidade de UAVs no início;

- CA: Percentual da cobertura territorial realizada pelos UAVs durante o tempo de simulação. Essa métrica é calculada a partir da divisão da área do ambiente emn sub-áreas quadradas de lado igual ao diâmetro do raio de percepção do UAV. Se a distância entre o UAV e o centro de qualquer subárea é menor ou igual ao raio de alcance do sensor de percepção, então essa sub-área é contabilizada. Ao final, é dividido a soma das sub-áreas contabilizadas pela área total do ambiente. Ao definir o parâmetro Número de iterações para recalcular a área de cobertura, a contagem é reiniciada;

- TT: Percentual de alvos rastreados pelos UAVs durante o tempo de simulação. Essa métrica é calculada dividindo a quantidade de alvos em rastreamento pela quantidade total de alvos.
A Tabela I contém os valores dos parâmetros do ambiente que foram mantidos constantes durante este experimento. Para a quantidade de UAVs foram utilizados os valores: $1,5,20,40,60$ e 80 .

A seguir se encontram os gráficos com os resultados do Experimento 1. A Figura 1 apresenta os resultados obtidos para as métricas de (a) alvos rastreados, (b) área de cobertura territorial e (c) colisões.

Analisando os dados na Figura 1 (a), o aumento do número de UAVs resulta numa quantidade maior de alvos rastreados. O mesmo pode ser observado em (b), onde a cobertura territorial aumenta de acordo com o aumento de UAVs. Em (c), a quantidade de colisões aumenta um pouco quando o número de UAVs é maior que 20 , mas ainda assim se mantém praticamente constante.

Os resultados obtidos no Experimento 1 indicam que o aumento no número de UAVs aumenta a efetividade do sistema no que diz respeito à quantidade de alvos rastreados e à área de cobertura territorial. Pode-se também concluir que múltiplos UAVs executam atividades de rastreamento e patrulha melhor que apenas um UAV.

\begin{tabular}{|c|c|}
\hline Parámetro & Valor \\
\hline Área do ambiente & $10.000 \mathrm{~m}^{2}$ \\
\hline Velocidade máxima dos UAVs & $5 \mathrm{~m} / \mathrm{s}$ \\
\hline Fator de inércia dos LAVs & 1 \\
\hline Aceleração máxima dos UAVs & $1.5 \mathrm{~m} / \mathrm{s}^{2}$ \\
\hline Aceleraçâo cognitiva dos UAVs & 1,0 \\
\hline Aceleração social dos UAVs & 3,0 \\
\hline Raio de extensão dos UAVs & $10,0 \mathrm{~m}$ \\
\hline Raio de alcance do sensor anti-colisào & $1.000 \mathrm{~m}$ \\
\hline Limiar do sensor do sensor anti-colisão & $100 \mathrm{~m}$ \\
\hline Segurança do sensor anti-colisāo & $300 \mathrm{~m}$ \\
\hline Raio de alcance do sensor de percepçāo & $1.000 \mathrm{~m}$ \\
\hline Limiar do sensor de percepção & $75,0 \mathrm{~m}$ \\
\hline Raio de alcance do sensor de comunicação & $1.000 \mathrm{~m}$ \\
\hline Limiar do sensor de comunicaçāo & $900,0 \mathrm{~m}$ \\
\hline Segurança do sensor de comunicação & $800,0 \mathrm{~m}$ \\
\hline Número máximo de comunicações & $2 m$ \\
\hline Quantidade de alvos & 5 \\
\hline Velocidade máxima dos alvos & $7,0 \mathrm{~m} / \mathrm{s}$ \\
\hline Aceleração máxima dos alvos & $1.5 \mathrm{~m} / \mathrm{s}$ \\
\hline Quantidade de obstáculos & 2 \\
\hline Raio de extensào dos obstáculos & $10,0 \mathrm{~m}$ \\
\hline Número de simulações paralelas & 30.0 \\
\hline Número de iteraçōes para recalcular a área de cobertura & 500 \\
\hline Número de iteraçōes & 100.000 \\
\hline
\end{tabular}

Tabela I

TABELA 1: PARÂMETROS MANTIDOS CONSTANTES NO CONJUNTO EXPERIMENTAL I.

\section{B. Conjunto experimental 2}

Este conjunto experimental tem como objetivo analisar o desempenho do modelo observando-se a métrica TT em função da velocidade relativa entre alvos e UAVs. Foram considerados 1,3 e 5 alvos. A seguir se encontram os gráficos com os resultados do Experimento 2. A Figura 2 apresenta a métrica TT em função da velocidade relativa 
entre alvos e UAVs para um alvo na Figura 2 (a), três alvos em (b) e cinco alvos em (c).

Analisando os dados na Figura 2, quando a velocidade relativa entre os alvos e UAVs é menor que 1 observa-se que a métrica TT mantém-se praticamente constante e próximo dos 100

Para cinco alvos foi observado um comportamento interessante: os UAVs competem entre si por um mesmo alvo e ignoram outros possíveis alvos no ambiente. Isso ocorre devido à modelagem do sistema, onde a velocidade dos UAVs tende à velocidade dos alvos. Como consequência, quanto mais lentos os alvos estiverem, mais lentos estarão os UAVs. Este problema pode ser resolvido com o uso de mecanismos colaborativos, levando os UAVs a não rastrearem os alvos que já estão sendo rastreados pelos vizinhos.

\section{Conjunto experimental 3}

Este conjunto experimental tem como objetivo analisar a eficácia do modelo observando-se a métrica TT em função do número de UAVs, com velocidade relativa entre alvos e UAVs igual a 0,8 . Foram considerados 1,3 e 5 alvos. Na Figura 3 se encontram os gráficos com os resultados do Experimento 3, com a métrica TT em função do número de UAVs para um (a), três (b) e cinco alvos (c).

(a) Um alvo

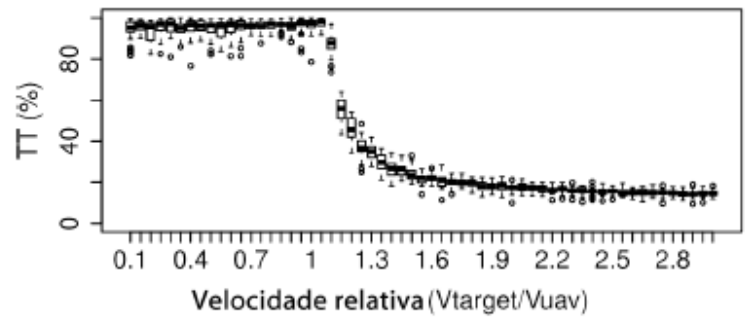

(b) Três alvos

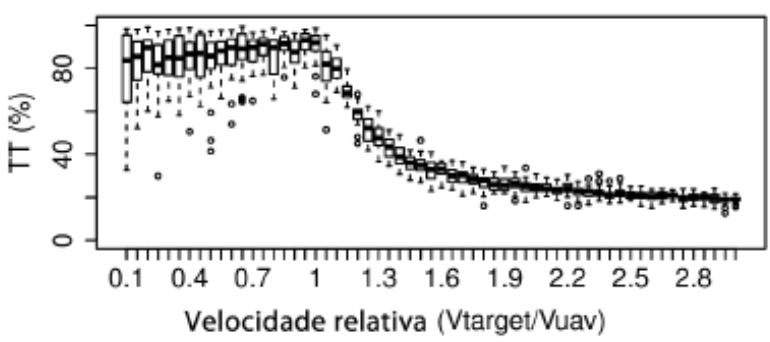

(c) Cinco alvos

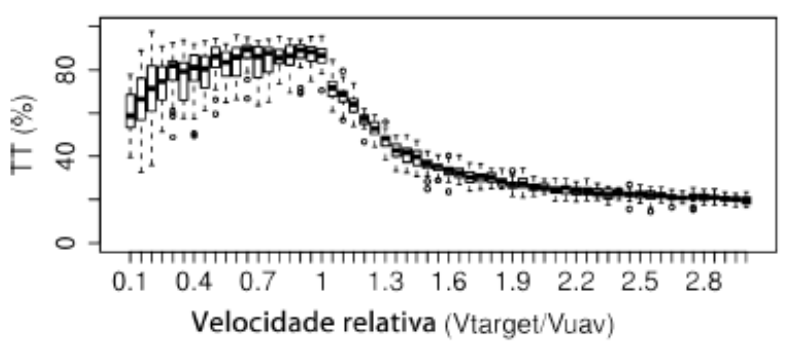

Figura 1. Experimento 1: Análise de CA (a), TT (b) e CL (c) em função do número de UAVs.

Analisando os dados na Figura 3, pode-se observar que 10 alvos já são suficientes para um rastreamento eficaz se houver apenas alguns alvos, porém serão necessários mais UAVs se o número de alvos for maior. Por exemplo, seriam necessários pelo menos 20 UAVs para um rastreamento eficaz de cinco alvos.

\section{Conclusões e Trabalhos Futuros}

Neste trabalho está sendo possível analisar e visualizar o comportamento coordenado de múltiplos UAVs utilizando inteligência de enxames em uma rede de comunicação ad hoc.

As estratégias e mecanismos adotados, juntamente com os resultados obtidos, evidenciam a possibilidade de avaliar previamente a utilização de UAVs em atividades de patrulha territorial e rastreamento de alvos móveis simultaneamente. Durante todas as simulações realizadas, nenhum UAV invadiu o espaço aéreo vizinho.

A utilização de inteligência de enxames e a topologia de comunicações ad hoc para coordenação dos UAVs provou

(a) Alvos rastreados

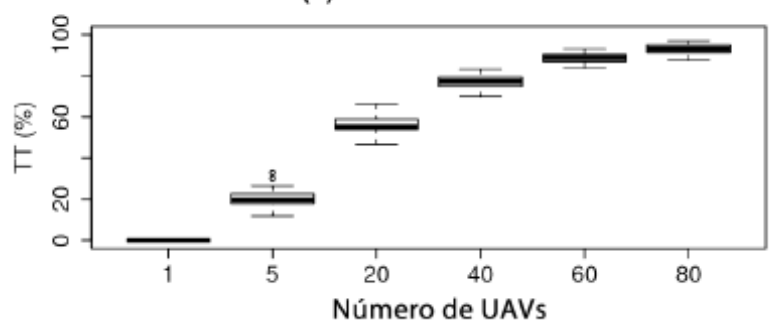


(b) Área de cobertura

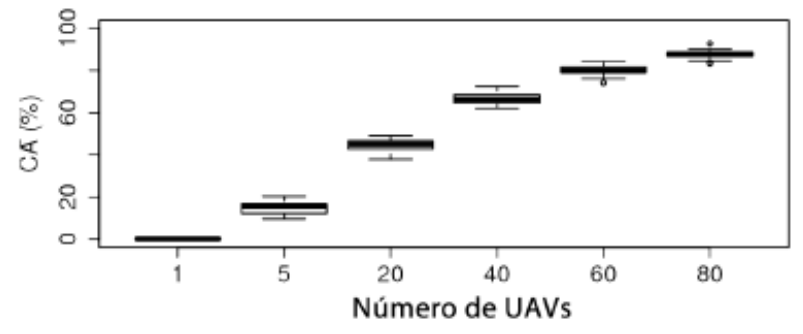

(c) Colisões

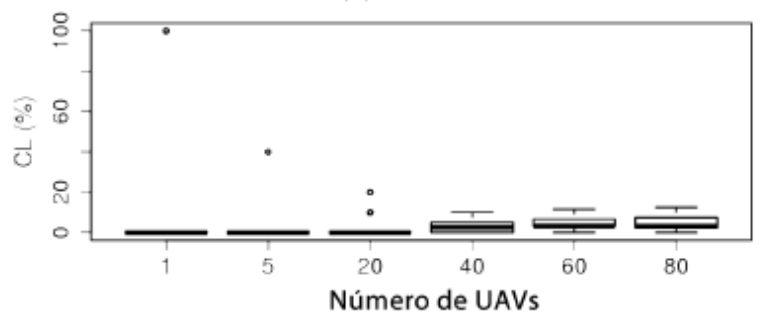

Figura 2. Experimento 2: Análise da quantidade do percentual de alvos rastreados (TT) em função da velocidade relativa entre os alvos e UAVs para um (a), três (b) e cinco alvos (c).

serem boas escolhas, indicando ser uma boa alternativa na resolução de problemas de cobertura e exploração territorial, controle distribuído, difusão e interação em sistemas multirobôs.

De forma específica, foi possível observar um comportamento não desejado de competição entre os UAVs quando a velocidade relativa 'e mais baixa, o que pode causar uma diminuição no percentual de alvos rastreados.

A análise dos resultados também indica a escalabilidade do sistema, pois a métrica CL não aumentou significativamente no intervalo de 1 a 80 UAVs, mas permaneceu praticamente estável e com baixo desvio padrão.

Para os trabalhos futuros fica a possibilidade de configurar as posições dos UAVs, dos alvos e dos obstáculos em posições configuráveis, pois no momento as posições destes no ambiente são geradas de maneira aleatória. Fica também como trabalho futuro o aprimoramento do estado de patrulha e rastreamento de modo a evitar a competição entre os UAVs por um mesmo alvo. (a) Um alvo

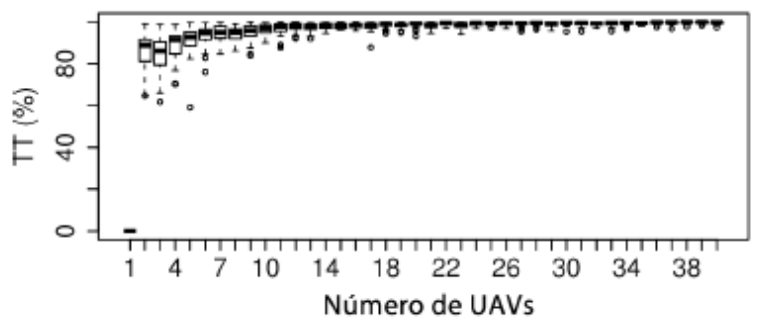

(b) Três alvos

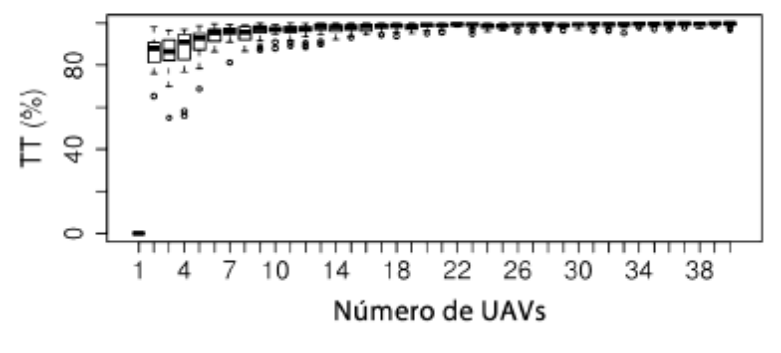

(c) Cinco alvos

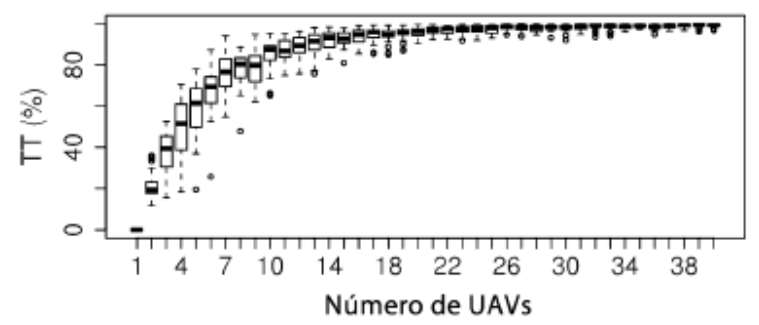

Figura 3. Figura 3: Análise do percentual de alvos rastreados (TT) em função do número de UAVs para um (a), três (b) e cinco alvos (c).

\section{Referências}

[1] BETHKE, B. Persistent Vision-Based Search and Track Using Multiple UAVs. Dissertação (Mestrado) - Massachusetts Institute of Technology, jun. 2007.

[2] GOEBEL, G. Unmanned Aerial Vehicles. Acessado em: Outubro de 2011. Disponível em: $<$ http://www.vectorsite.net/twuav.html $>$.

[3] ENGELBRECHT, A. P. Computational Intelligence An Introduction. 1. ed. West Sussex, Inglaterra: Wiley, 2002.

[4] KENNEDY, J.; EBERHART, R.; SHI, Y. Swarm Intelligence. 1. ed. San Francisco, Calif'ornia, USA: Morgan Kaufmann Publishers, 2001. 
[5] KENNEDY, J.; EBERHART, R. Particle swarm optimization. In: Proceedings of IEEE International Conference on Neural Networks. Perth, WA, Australia: IEEE, 1995. v. 4, p. 1942-1948.

[6] PORTELA, M. Simulação de robôs aquáticos utilizando inteligência de enxames para inspeção de dutos submarinos.

2009. 67 p. Trabalho de conclusão de curso - Engenharia da Computação, UPE, Recife-PE.

[7] SINSLEY, G. et al. An intelligent controller for collaborative unmanned air vehicles. In: IEEE Symposium on Computational Intelligence in Security and Defense Applications. Honolulu, HI: IEEE, 2007. p. 139-144.

[8] J. P. How, E. K.; KUWATA, Y. Flight demonstrations of cooperative control for uav teams. In: Proceedings of 3rd Unmanned Unlimited Technical Conference, Workshop and Exhibit. Chicago, IL: AIAA, 2004. p. 505-513. 